\title{
IDADE FISIOLÓGICA DOS OVOS DE AEDES (STEGOMYIA) AEGYPTI (LINNAEUS, 1762) (DIPTERA, CULICIDAE)
}

\author{
Heloisa Helena Garcia da Silva ${ }^{*}$, Ionizete Garcia da Silva ${ }^{* *}$, Carmeci Natalina \\ Elias $^{* * *}$, Sandro Pinheiro Souza Lemos ${ }^{* * *}$, Andréa Pereira Rocha
}

\section{RESUMO}

Ovos de Aedes (Stegomyia) aegypti (Linnaeus, 1762) (Diptera, Culicidae) foram colocados para incubar, numa bacia com água, após um período de 121 dias da oviposição, numa câmara climatizada a $28 \pm 1^{\circ} \mathrm{C}$, umidade relativa de $80 \pm 5 \%$ e fotofase de 12 horas. Os ovos permaneceram na água por dois meses e deram origem a cinco ciclos. Estes ciclos apresentavam diferenças significativas apenas quanto ao período de incubação dos ovos, nos quais as larvas de primeiro estádio eclodiam em grupos, mostrando que a fase embrionária apresentava idades fisiológicas significativamente diferentes entre si. A incubação média dos ovos de $A$. aegypti foi de 0,$9 ; 5,6 ; 33,0 ; 42,5$ e 53,2 dias, respectivamente, aos $1^{\circ}, 2^{\circ}, 3^{\circ}, 4^{\circ}$ e $5^{\circ}$ ciclos. Este estudo teve a finalidade de compreender melhor a biologia e o comportamento de $A$. aegypti servindo de orientação para ensaios biológicos de laboratório, bem como fornecer informações que auxiliem no planejamento das ações de controle.

UNITERMOS: Aedes aegypti. Desenvolvimento. Incubação. Dengue. Vetor.

\section{INTRODUÇÃO}

Aedes aegypti é um mosquito bem adaptado ao ambiente urbano ${ }_{1,2}^{2}$, com hábitos marcadamente antropofílico e sinantrópicos ${ }^{1,2}$, desenvolve-se em coleções d'água não poluída, em criadouros, que, em grande parte, são provenientes do lixo, da sociedade moderna, que não teve destino adequado e de ações antrópicas no meio ambiente.

- Farmacêutica do Depto. de Parasitologia do IPTSP/UFG.

* Prof. do Depto. de Parasitologia do IPTSP/UFG.

*.* Técnica da Fundação Nacional de Saúde - Goiânia.

*.*. Bolsista de Iniciação Tecnológica/RHAE.

***** Bolsista de Iniciação Científica/CNPq.

Recebido para publicação em 18/09/95 
SIIVA, HH.G : SILVA, IG.; ELIAS, CN. LEMOS, S.P.S. \& ROCHA, A.P. Idade fisiológica dos ovos de Aedes (Stegomyia) aegypti (Linnaeus, 1762) (Diptera, Culicidae). Rev. Pat. Trop.24 ( 2 ): 269 - 273, jul/dez. 1995 ,

Este trabalho teve a finalidade de esclarecer o ritmo de desenvolvimento da fase embrionária de $A$. aegypti, em laboratório, bem como, orientar ensaios biológicos 3,4 com esse mosquito e fornecer informações que subsidiem o planejamento das ações de controle.

\section{MATERIAL E MÉTODOS}

Origem dos mosquitos - capturaram-se larvas e pupas de A. aegypti em vários bairros de Goiânia, que foram levadas para o laboratório para completar o ciclo, e, destas, obtiveram-se adultos, que deram origem à série em estudo.

Câmara biológica - uma câmara foi projetada e construída ${ }^{3}$, tendo em atenção a biologia de $A$. aegypti, bem como a segurança para impedir a fuga dos insetos. Esta câmara foi climatizada a $28 \pm 1^{\circ} \mathrm{C}$, com umidade relativa de $80 \pm 5 \%$, e fotofase natural, cerca de 12 horas.

Incubação - após 121 dias da oviposição, colocou-se uma cartela com 749 ovos de $A$. aegypti, imerso em 1,5 litros de água de torneira (do sistema público de abastecimento), numa bacia esmaltada medindo $30 \mathrm{~cm}$ de diâmetro por $7 \mathrm{~cm}$ de altura, que era mantida na câmara biológica.

Alimentação - imediatamente após a eclosão, ofereceu-se alimentação às larvas. Diariamente, colocou-se em cada frasco, ração para gato, após ter sido triturada no gral com pistilo e coada em tela fina até se obterem finíssimas partículas. As fêmeas foram alimentadas em camundongos albinos empastelados ${ }^{3}$ e, os machos, em algodão(do tipo O.B.) embebido em água açucarada (na proporção de $12 \mathrm{~g}$ de açúcar cristal por $250 \mathrm{ml}$ de água)

Larvas, pupas e adultos - após a eclosão, as larvas foram individualizadas em frascos de 4,0 x 4,7 cm, permanecendo nestes até a emergência do adulto. No estágio de pupa, os frascos eram colocados dentro de uma gaiola para emergir os adultos, posteriormente, procedía-se à determinação do sexo e ao acasalamento.

\section{RESULTADOS E DISCUSSÃO}

Dos 749 ovos de $A$. aegypti colocados para incubar, eclodiram 713 larvas, agrupadas em cinco períodos definidos de incubação que variaram de 0,9 a 53,2 dias, apresentando uma fertilidade de $95,19 \%$. A duração média do ciclo evolutivo de $A$
SILVA, H.H.G.; SILVA, I.G.; ELIAS, C.N.; LEMOS, S.P.S. \& ROCHA, A.P. Idade fisiológica dos ovos de Aedes (Stegomyia) aegypti (Linnaeus, 1762) (Diptera, Culicidae). Rev. Pat. Trop.24 ( 2 ): 269 - 273, jul/dez. 1995.

aegypti foi de 10,$0 ; 13,6 ; 41,4 ; 50,3 ; 61,5$ dias, respectivamente, aos $1^{\circ}, 2^{\circ}, 3^{\circ}, 4^{\circ} \mathrm{e}$ $5^{\circ}$ ciclos.

Constatou-se diferença significativa entre todos os períodos de incubação, pela análise da variância, ao nível de $1 \%$. Basicamente, os ciclos foram diferentes entre si, pela fase embrionária, que apresentou idades fisiológicas diferentes na incubação dos ovos.

Tabela 1. Duração dos períodos de incubação, dos estádios larvais e estágio de pupa de Aedes aegypti, à temperatura de $28^{\circ} \mathrm{C}$ e umidade relativa de $80 \%$.

\begin{tabular}{lccccc}
\hline & \multicolumn{5}{c}{ CICLO EVOLUTIVO EM DIAS } \\
\cline { 2 - 6 } PERÍODOS & $1^{\circ}$ & $2^{\circ}$ & $3^{\circ}$ & $4^{\circ}$ & $5^{\circ}$ \\
\hline INCUBAÇÃO & $0,9 \pm 0,09$ & $5,6 \pm 0,10$ & $33,0 \pm 0,01$ & $42,5 \pm 0,15$ & $53,2 \pm 0,1$ \\
LARVA -1 & $2,4 \pm 0,21$ & $1,2 \pm 0,08$ & $1,7 \pm 0,01$ & $1,3 \pm 0,03$ & $1,1 \pm 0,03$ \\
LARVA -2 & $0,9 \pm 0,07$ & $0,8 \pm 0,06$ & $1,0 \pm 0,01$ & $1,1 \pm 0,10$ & $1,5 \pm 0,10$ \\
LARVA -3 & $1,2 \pm 0,07$ & $1,0 \pm 0,02$ & $1,0 \pm 0,01$ & $1,0 \pm 0,10$ & $0,9 \pm 0,03$ \\
LARVA -4 & $2,6 \pm 0,13$ & $3,0 \pm 0,18$ & $2,5 \pm 0,07$ & $2,5 \pm 0,10$ & $2,7 \pm 0,14$ \\
PUPA & $2,0 \pm 0,03$ & $2,1 \pm 0,10$ & $2,2 \pm 0,06$ & $2,1 \pm 0,10$ & $2,3 \pm 0,08$ \\
\hline CICLO & $10,0 \pm 0,23$ & $13,6 \pm 0,20$ & $41,4 \pm 0,08$ & $50,3 \pm 0,18$ & $61,5 \pm 0,18$ \\
\hline
\end{tabular}

A idade fisiológica dos ovos de $A$. aegypti foi avaliada após um período de 4 meses da oviposição, mantidos em recipientes plásticos numa câmara biológica. $\mathrm{A}$ fase de ovo ${ }^{6}$ é a etapa mais importante do desenvolvimento desse mosquito, resiste a prolongados períodos de dormência, apresenta a incubação com diferentes idades embriogênicas, isto tem garantido ao $A$. aegypti sua manutenção na natureza, durante variações climáticas sazonais. A idade fisiológica dos ovos deve ser um importante mecanismo que o $A$. aegypti se utiliza para colocar, em diferentes períodos de cada mês ou de uma estação do ano, uma população de adultos e, desta forma, manter a densidade do mosquito sem grandes flutuações.

A temperatura de $28 \pm 1^{\circ} \mathrm{C}$, umidade relativa de $80 \pm 5 \%$ e fotofase de 12 horas, mostraram-se favoráveis ao desenvolvimento de $A$. aegypti. Das 713 larvas eclodidas, apenas 13 morreram durante a realização dos ciclos evolutivos, isto 
SILVA, H.H.G.; SILVA, I.G.; ELIAS, C.N.; LEMOS, S.P.S. \& ROCHA, A.P. Idade fisiológica dos ovos de Aedes (Stegomyia) aegypti (Linnaeus, 1762) (Diptera, Culicidae). Rev. Pat. Trop.24 ( 2 ): 269 - 273, jul/dez. 1995.

representa uma mortalidade de $1,8 \%$. Como estas condições climáticas são similares às das estações de verão e outono em Goiânia, pode-se compreender o porquê do adensamento do $A$. aegypti nestas épocas do ano e as ocorrências coincidentes, nos dois últimos anos (1994/95), das duas epidemias de dengue.

\section{SUMMARY}

\section{Physiological age of Aedes (Stegomyia) aegypti eggs (Linnaeus, 1762) (Diptera,} Culicidae)

121 days after oviposition, Aedes (Stegomyia) aegypti (Linnaeus, 1762) (Diptera, Culicidae) eggs were placed in a warm water bath, in a temperature controlled chamber $\left(28 \pm 1^{\circ} \mathrm{C}\right), 80 \pm 5 \%$ humidity and a 12 hours photoperiod. The eggs originated 5 cycles, in a period of 2 months. The incubation period, was the only variation. The first instar hatching was in groups, showing that the embrionic phase presented significant differences. The average incubation time was $0.9 ; 5.6$; $33.0 ; 42.5$ and 53,2 , at the $1^{\text {st }}, 2^{\text {nd }}, 3^{\text {rd }}, 4^{\text {th }}, 5^{\text {th }}$ cycles. The purpose of this study was a better understanding of the biology and behaviour of $A$. aegypti, for a further use in other laboratory experiments and also to provide valuable information on control actions.

KEYWORDS: Aedes aegypti. Development. Incubation. Dengue. Vector

\section{REFERÊNCIAS BIBLIOGRÁFICAS}

1.SILVA, I.G.da; ARAUJO, E.S. de O.; SILVA, H.H.G. da; CANTUÁRIA, P.B. Ocorrência de Aedes (Stegomyia) aegypti (Linnaeus, 1762) (Diptera, Culicidae) em Goiânia. An.Soc.Ent.Brasil, 20:459-460, 1991.

2.SILVA, I.G.da; CANTUÁRIA, P.B.; SILVA, H.H.G.da \& ARAÚJO, E.S. de O. Distribuição de Aedes (Stegomyia) aegyti (Linnaeus, 1762) (Diptera, Culicidae) em Goiânia. Rev.Pat.Trop., 20:1-5, 1991

3.SILVA, I.G.da; CAMARGO, M.de F.; ELIAS, C.N.; ISAC, E. \& SANTOS, A.H. Metodologia de criação de Aedes (Stegomyia) aegypti Linnaeus, 1762)
SILVA, H.H.G.; SILVA, I.G.; ELIAS, C.N.; LEMOS, S.P.S. \& ROCHA, A.P. Idade fisiológica dos ovos de Aedes (Stegomyia) aegypti (Linnaeus, 1762) (Diptera, Culicidae). Rev. Pat. Trop.24 ( 2 ): 269 - 273, jul/dez. 1995.

(Diptera, Culicidae), em condições de laboratório. Rev.Goiana Med., 39:2326, 1994.

4.SILVA, I.G. da; CAMARGO, M. de F.; ELIAS, M. \& ELIAS, C.N. Ciclo evolutivo de Aedes (Stegomyia) aegypti (Linnaeus, 1762) (Diptera, Culicidae). Rev.Pat.Trop.,22:43-48, 1993.

5.SILVA, I.G.da; CAMARGO, M. de F.; GUIMARÃES, F.L.; ELIAS, M. \& OLIVEIRA, A.W.S. Estudo da eficácia da Deltametrina (K-Othrine UBV 0,4\% e 1\%) no combate ao Aedes (Stegomyia) aegypti (Linnaeus, 1762) e ao Culex quinquefasciatus (Wiedmann, 1828) (Diptera, Culicidae). Rev.Pat.Trop., 22:49-56, 1993

6.THOMAS, H.D. Preliminary studies on the physiology of Aedes aegypti (Diptera, Culicidade). I. The hactching of eggs under sterile conditions.J.Parasit., 29:324-327, 1943. 